\title{
Effect of Patient Counselling Based Intervention in Hypertensive Outpatients
}

\author{
Parshwa Shah, Het Patel, Kanjav Oza, Nupoor Shah, Shrikalp Despande, Chintan Patel* \\ Department of pharmacology and pharmacy practice, K. B. Institute of Pharmaceutical Education and Research, \\ Gandhinagar, Gujarat, INDIA.
}

\begin{abstract}
Objectives: To evaluate the effectiveness of patient counselling based intervention on blood pressure, medication adherence and knowledge in hypertensive patients. Materials and Methods: A prospective, multi-centric, randomized controlled interventional study conducted for period of 4 months at 2 outpatient's clinics in Gandhinagar city, Gujarat. Enrolled patients were randomized into 2 groups (Intervention and Control) using simple randomization technique with allocation ratio of $1: 1$. Morisky Medication Adherence Scale (MMAS-4), self-designed Case Record Form (CRF) and self-designed knowledge questionnaire were utilised for data collection. Each patient was followed for 2 months with a total of 4 follow-ups each 15 days apart. Patient counselling and education was provided using patient information leaflet (PIL) in interventional group. Results: A total of 105 patients were randomized into two groups. The result revealed significant reduction i.e. $10 \mathrm{mmHg}$ in systolic blood pressure (SBP) and $5.78 \mathrm{mmHg}$ in diastolic blood pressure (DBP) in interventional group $(p<0.0001)$ from baseline to final visit whereas no significant difference was seen in control group. Interventional group showed significant change in medication adherence $(p=0.0011)$ as well as in hypertension related knowledge $(p<0.0001)$ wherein control group showed no significant change at the end of study. Conclusion: It is to be concluded that patient counselling based intervention using PIL improved blood pressure and medication adherence in hypertensive patients. The intervention was helpful in enhancing knowledge and creating awareness regarding disease and medication.
\end{abstract}

Key words: Hypertension, Pharmacist intervention, Medication adherence, Blood pressure, Hypertension related knowledge.

\section{INTRODUCTION}

Hypertension, a persistence increase in blood pressure is one of the most common chronic disease and an important health problem worldwide. ${ }^{1} \mathrm{t}$ is one of the basic avoidable risk factor for cardiovascular disease having a disturbing effect on people's health and results in needless morbidity and mortality. ${ }^{2}$ The disease has been appropriately nicknamed the silent killer because most patients with hypertension have no symptoms, and therefore the disease often goes undetected. For the treatment of hypertension, guidelines and evidence based management have been made available, however blood pressure control still remains a big challenge for healthcare providers. Control of hypertension results in better long term health outcomes. Health benefits of controlled blood pressure can be achieved by use of multidisciplinary physician-pharmacist collaborative work. Along with physicians and other health care professionals, clinical pharmacist has indulged themselves in the process of healthcare awareness. Clinical pharmacist recommendation for alteration in pharmacotherapy and patient counselling has significantly improved medication adherence. Clinical pharmacist has complimented physicians in the management of hypertensive patient in improving antihypertensive medication adherence and
DOI: 10.5530/ijopp.15.1.5

Address for correspondence: Dr. Chintan Patel, Department of Pharmacology and Pharmacy Practice, K. B. Institute of Pharmaceutical Education and Research, Gandhinagar, Gujarat, INDIA Email id: kbiper.research2020@gmail.com

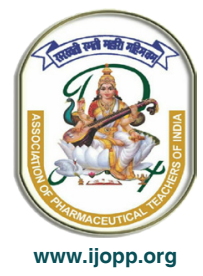


reducing systolic and diastolic blood pressure. ${ }^{3} \mathrm{~A}$ pharmacist can contribute in positive outcome by educating and counselling patient to prepare and motivate them for their Pharmacotherapeutics regimen. ${ }^{4}$ Patient counselling helps in providing medication information and advising them about diet and lifestyle modification. This involves one to one interaction between pharmacist, patients and or caregiver. ${ }^{5}$ Patient Information Leaflets (PILs) are produced for the benefits of patient and are globally accepted as a tool to motivate patients and the use of pictorials enhances the patients and liberalize the understanding about their disease and the motion to cure it. ${ }^{4}$

Patients with hypertension may fail to follow their medication because of symptomless nature of their condition, long duration of therapy, side effects, complicated drug regimens, lack of understanding about hypertension management as well as lack of patient education could contribute significantly to noncompliance. ${ }^{6,7}$ According to the National Family Health Survey (NFHS) 2019-20, Gujarat reported 20.3\% male and $20.6 \%$ female having elevated blood pressure or taking medication to control blood pressure.

Several studies have reported that hypertensive patients are benefited from pharmacist intervention which focus on improving the knowledge regarding the condition, improvement in medication adherence and various life style modification. So, this study was aimed to evaluate to effect of patient counselling on improvement in blood pressure and medication adherence in hypertensive outpatients. The study was first of its kind in Gandhinagar city, Gujarat.

\section{MATERIALS AND METHODS}

\section{Study design and Location}

A multi-centric, prospective, randomized controlled interventional study carried out at Pagrav multispeciality hospital and Sanjeevani heart and medical hospital located in sector-23 and sector-21 respectively in Gandhinagar, Gujarat.

\section{Study population}

Patients who visited the hospital setting were screened and the subjects were selected according to the inclusion/ exclusion criteria.

\section{Sample size}

Sample size was calculated to be 105 after considering $10 \%$ non-response rate by collecting data from previous published literature.

\section{Eligibility criteria}

Inclusion Criteria: Patient age more than 18 years of age, Hypertensive patient with or without comorbid conditions, Patient on antihypertensive medication for at least 1 month.

Exclusion Criteria: Patient with dementia or other psychiatric conditions, Pregnant or lactating women, Patient with heart failure, stroke, end stage renal disease and end stage liver disease.

\section{Study duration}

Total duration of study was 6 months; 4 months data collection and 2 months data analysis.

\section{Study Material}

Prior to collection of data, written consent was taken from patient using Informed Consent Form designed in English as well as Gujarati. The demographic details was collected in Case Record Form. Medication adherence was assessed using morisky medication adherence scale (MMAS-4) and the scale was translated into regional language (Gujarati). The translated questionnaire was validated. On the basis of score the patients were classified into three categories: High (Score of 4), Medium (Score of 2 or 3), Low (Score of 1). Selfdesigned and validated patient information leaflet was provided in both languages (English and Gujarati) which consisted of following points: 1. What is hypertension? 2. Lifestyle modifications. 3. Risk factor. 4. Complications. 5. Normal range of blood pressure. Self-designed and validated knowledge questionnaire was used to assess hypertension related knowledge which consisted of 15 questions assessing knowledge in following areas: normal value, risk factors, sign and symptoms, lifestyle modification and medication, complications. Score between 24 to 30 was categorise as high knowledge, between 15 to 24 as medium knowledge and less than 15 as low knowledge. The questionnaire was translated into regional language (Gujarati). The translated questionnaire was validated.

\section{Study procedure}

\section{Approval}

\section{Ethics Committee Approval}

Prior to the study, it was necessary to take the approval/ permission from the ethics committee of KBIPER, KSV University to conduct the following study in the below mentioned hospitals of Gandhinagar. 


\section{Data collection procedure}

Patient were first screened according to the inclusion and exclusion criteria and eligible patient were explained regarding the study procedure and informed consent was taken prior to the enrolment of patient in study. Enrolled patient were than randomized into one of the two groups (Intervention and control) with simple randomisation technique in allocation ratio 1:1. Those in intervention group received various pharmacist intervention which included counselling regarding medication adherence, education regarding hypertension and patient information leaflet was explained and provided to patients randomised in intervention group whereas those in control group did not receive any such intervention.

At BASELINE VISIT (clinic visit), demographic details were noted. Patient's baseline blood pressure was measured and were assessed for medication adherence and knowledge. Those patients in intervention group were educated and counselled regarding hypertension, lifestyle modifications, medicine and importance of adherence. At FIRST VISIT (clinic/telephone visit- 15 days +/- 1 day from baseline visit), Patients blood pressure was measured and counselling was provided. At SECOND VISIT (clinic visit- 30 days $+/-1$ day from baseline visit), Patients blood pressure was measured. Those patients in intervention group were followed up regarding hypertension related knowledge and importance of adherence and the patient was counselled. At THIRD VISIT (clinic/telephone visit- 45 days +/- 1 day from baseline visit), Patient blood pressure was measured and counselling was provided. At FINAL VISIT (clinic visit- 60 days $+/-1$ day from baseline visit), Patients final blood pressure was measured and were assessed for medication adherence and knowledge. The conceptual framework of study is illustrated in Figure 1.

\section{Data management and statistical analysis}

Data management and analysis was conducted using Microsoft excel 2013 (v15.0) and Graph Pad Prism version 5.01 for windows, Graph Pad Software, La Jolla California USA. A two tailed $P$ value $<0.05$ was considered to be statistically significant for all analysis. Normality test of data was conducted using ShapiroWilk test and on the basis of result obtained appropriate statistical test (Parametric and Non-parametric) was selected for statistical analysis of data. Descriptive statistic was used for analysis of demographic details of study population. As systolic and diastolic blood pressure data was not normally distributed, nonparametric test i.e. Friedman test was used as there was comparison between three matched groups. Chi-square

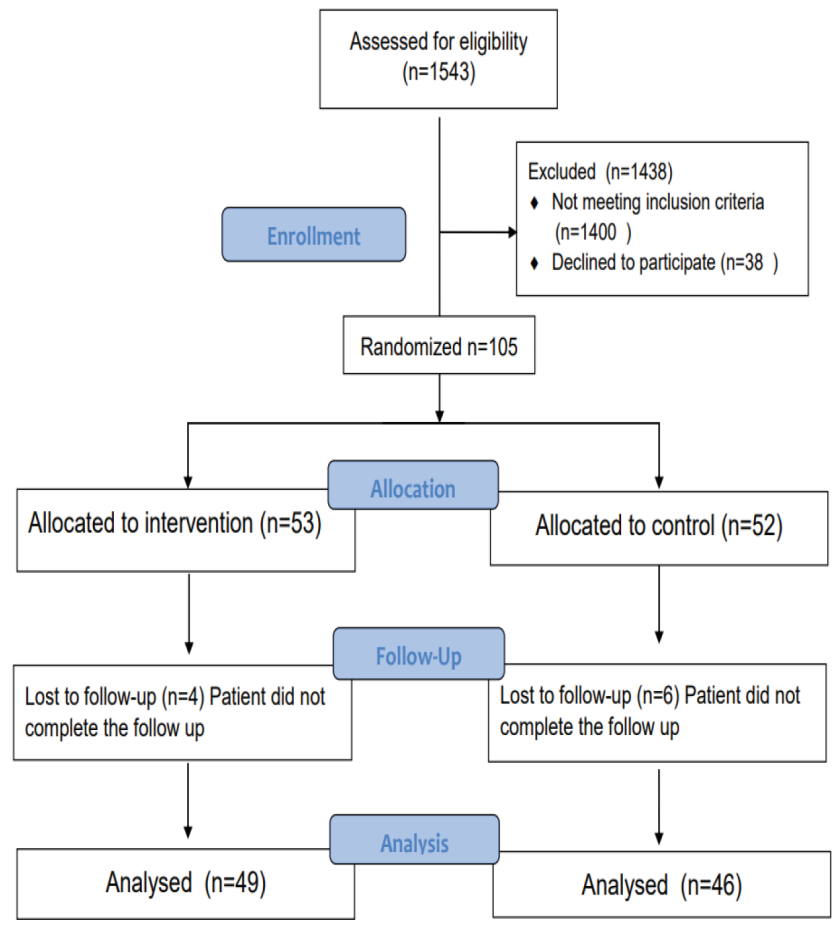

Figure 1: Conceptual Framework of Study.

test was used to compare the result of medication adherence and hypertension related knowledge.

\section{RESULTS}

Table 1 describes the demographic details of study population in which majority of them belong to age group of 50-59 followed by 60-69. Male were predominately higher than female. $47.6 \%$ were over-weight and maximum number of patients had hypertension since more than 5 years. Half of the total patients had home blood pressure monitoring device and majority (89.5\%) had no significant social history. Diabetes mellitus was the most commonly occurring comorbid condition among individuals and $60 \%$ from total patients had history of hypertension running in their family.

Table 2 shows assessment of change in systolic as well as diastolic blood pressure among study patients in both intervention and control group form baseline to final visit. Baseline mean \pm SD systolic blood pressure in intervention group was $145.3 \pm 18.01$ whereas in control group it was $138.5 \pm 14.40$. The mean change in blood pressure was $10.7 \mathrm{mmHg}$ in intervention group and $0 \mathrm{mmHg}$ in control group. According to the $\mathrm{p}$ value intervention group showed statistically significant change in SBP compared to control group. Baseline mean \pm SD diastolic blood pressure in intervention group was $92.31 \pm 9.61$ whereas in control group it was 


\section{Table 1: Demographic Details of study population.}

No. of participants

Intervention $n=53(\%) \quad$ Control $n=52(\%)$

Total $n=105(\%)$

\begin{tabular}{|c|c|c|c|}
\hline \multicolumn{4}{|l|}{ Age } \\
\hline $30-39$ & $2(3.7)$ & $1(1.9)$ & $3(2.8)$ \\
\hline $40-49$ & $9(16.9)$ & $9(17.3)$ & $18(17.1)$ \\
\hline $50-59$ & $16(30.1)$ & $14(26.9)$ & $30(28.5)$ \\
\hline $60-69$ & $16(30.1)$ & $11(21.1)$ & $27(25.7)$ \\
\hline $70-79$ & $10(18.8)$ & $14(26.9)$ & $24(22.8)$ \\
\hline $80-89$ & $0(0)$ & $3(5.7)$ & $3(2.8)$ \\
\hline \multicolumn{4}{|l|}{ Gender } \\
\hline Male & $26(49.0)$ & $32(61.5)$ & $58(55.2)$ \\
\hline Female & $27(50.9)$ & $20(38.4)$ & $47(44.7)$ \\
\hline \multicolumn{4}{|l|}{ BMI Category } \\
\hline Under-weight & $2(3.7)$ & $1(1.9)$ & $3(2.8)$ \\
\hline Normal & $14(26.4)$ & $21(40.3)$ & $35(33.33)$ \\
\hline Over-weight & $32(60.3)$ & $18(34.6)$ & $50(47.6)$ \\
\hline Obese & $4(7.5)$ & $10(19.2)$ & $14(13.3)$ \\
\hline Extreme Obesity & $1(1.8)$ & $2(3.8)$ & $3(2.8)$ \\
\hline \multicolumn{4}{|l|}{ Duration of disease } \\
\hline$<1$ Year & $5(9.4)$ & $4(7.6)$ & $9(8.5)$ \\
\hline 1-3 Years & $10(18.8)$ & $15(28.8)$ & $25(23.8)$ \\
\hline 3-5 Years & $11(20.7)$ & $3(5.7)$ & $14(13.3)$ \\
\hline$>5$ Years & $27(50.9)$ & $30(57.6)$ & $57(54.2)$ \\
\hline \multicolumn{4}{|l|}{ Home BP Device } \\
\hline Yes & $24(45.2)$ & $23(44.2)$ & $47(44.7)$ \\
\hline No & $29(55.7)$ & $29(55.7)$ & $58(55.2)$ \\
\hline \multicolumn{4}{|l|}{ Social History } \\
\hline Yes & $3(5.6)$ & $8(15.3)$ & $11(10.4)$ \\
\hline No & $50(94.3)$ & $44(84.6)$ & $94(89.5)$ \\
\hline \multicolumn{4}{|l|}{ Comorbid Condition } \\
\hline Present & $23(43.3)$ & $27(51.9)$ & $50(47.6)$ \\
\hline Absent & $30(56.6)$ & $25(48.0)$ & $55(52.3)$ \\
\hline \multicolumn{4}{|l|}{ Type of comorbidities } \\
\hline DM & 11 & 11 & 22 \\
\hline Hypercholesterolemia & 1 & 3 & 4 \\
\hline Hypothyroidism & 4 & 5 & 9 \\
\hline DM + Hypothyroidism & 2 & 0 & 2 \\
\hline DM + Hypercholesterolemia & 0 & 1 & 1 \\
\hline DM + Hypothyroidism + Hypercholesterolemia & 0 & 1 & 1 \\
\hline Hypothyroidism + Hypercholesterolemia & 3 & 1 & 4 \\
\hline Other & 2 & 5 & 7 \\
\hline \multicolumn{4}{|l|}{ Family History } \\
\hline Yes & $34(64.1)$ & $26(50.0)$ & $60(57.1)$ \\
\hline No & $19(35.8)$ & $26(50.0)$ & $45(42.8)$ \\
\hline
\end{tabular}


Table 2: Assessment of change in Blood Pressure.

\begin{tabular}{|c|c|c|c|c|}
\hline SYSTOLIC BP & Mean(SD) in $\mathrm{mmHg}$ & Median in $\mathrm{mmHg}$ & Friedman value $^{a}$ & $p$ value $^{b}$ \\
\hline \multicolumn{5}{|c|}{ INTERVENTION $n=49$} \\
\hline Baseline & $145.3(18.01)$ & 140 & 28.69 & $<0.0001^{*}$ \\
\hline Second visit & $135.5(10.62)$ & 132 & & \\
\hline Final visit & $134.6(10.12)$ & 132 & & \\
\hline \multicolumn{5}{|l|}{ CONTROL $n=46$} \\
\hline Baseline & $138.5(14.40)$ & 140 & 1.086 & 0.5811 \\
\hline Second visit & $138.2(12.20)$ & 138 & & \\
\hline Final visit & $138.5(11.96)$ & 140 & & \\
\hline DIASTOLIC BP & Mean(SD) in $\mathrm{mmHg}$ & Median in $\mathrm{mmHg}$ & Friedman value $^{a}$ & $p$ value $^{b}$ \\
\hline \multicolumn{5}{|c|}{ INTERVENTION $n=49$} \\
\hline Baseline & $92.31(9.618)$ & 90 & 16.77 & $<0.0001^{*}$ \\
\hline Second visit & $86.41(7.024)$ & 88 & & \\
\hline Final visit & $86.53(5.193)$ & 88 & & \\
\hline \multicolumn{5}{|l|}{ CONTROL $n=46$} \\
\hline Baseline & 87.33(9.888) & 90 & 0.5478 & 0.7604 \\
\hline Second visit & $88.24(5.952)$ & 90 & & \\
\hline Final visit & $88.74(7.123)$ & 90 & & \\
\hline
\end{tabular}

a- Friedman statistic value, b- $p$ value obtained from Friedman statistic, *- Statistically significant.

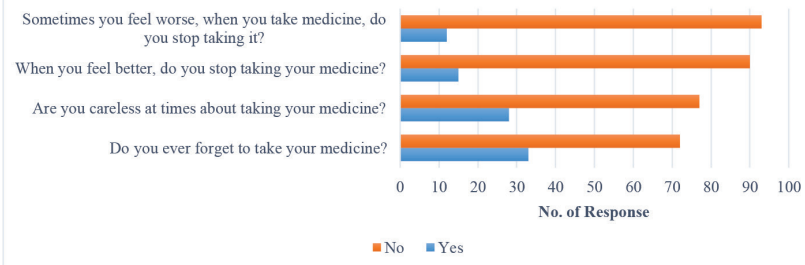

Figure 2: Response to morisky medication adherence scale (MMAS-4).

87.33 \pm 9.88 . The mean change in blood pressure was $5.78 \mathrm{mmHg}$ in intervention group and $-1.41 \mathrm{mmHg}$ in control group. According to the $p$ value intervention group showed statistically significant change in DBP compared to control group.

The following bar graph in Figure 2 depicts that the most common reason for medication non-adherence was forgetfulness $(31.4 \%)$ followed by carelessness (26.6\%). The total number of patient in intervention group were 49 out of which $44.89 \%$ were non-adherent and out of 46 patient in control group 34.7\% were non-adherent at baseline. At final visit it was found that $87.7 \%$ patient were adherent in intervention group and $63.0 \%$ in control group. According to the $\mathrm{p}$ value intervention group showed statistically significant change in medication adherence compared with control group as indicated in Table 3.
Table 4 illustrates the assessment of change in hypertension related knowledge. The percentage of patient in intervention group i.e. 57.1\% were having poor knowledge regarding hypertension whereas $60.8 \%$ in control at baseline. At final visit it was found that $89.7 \%$ patient were having good knowledge in intervention group and $43.4 \%$ in control group. According to the $p$ value intervention group showed statistically significant change in hypertension related knowledge compared with control group from baseline to end of study.

\section{DISCUSSION}

$55.2 \%$ patients in the study were male, which was higher than female. This value do not differ greatly from data reported by several other studies, as hypertension being more prevalent in male compared to female. ${ }^{1,2,4}$ In this study, the majority of patients were overweight and the maximum no. of patients were in the age group of 50-59. The findings were similar with the data reported by Sunita Parma et al. ${ }^{4}$ Male gender, age $>55$ years and $\mathrm{BMI}>30 \mathrm{~kg} / \mathrm{m}^{2}$ are commonly occurring risk factor for development of hypertension due to which maximum no. of patient were in these category. Patient enrolled in our study had no significant social habits (Alcohol, smoking and tobacco). Diabetes mellitus was most commonly occurring comorbid condition amongst study patients. Majority of study patients had 
hypertension from $>5$ years which was similar with the findings of study reported by Fahad Saleem et al. ${ }^{8}$

We focused primarily on SBP because studies have shown that SBP is more valuable in predicting the risk of cardiovascular disease than DBP, especially in middle-aged, diabetic, and older patients. Pharmaceutical intervention have been shown to improve blood pressure in previously published studies. ${ }^{1-3}$ Study conducted by Pei-Xi Zhao et al. ${ }^{1}$ showed $8.5 \mathrm{mmHg}$ of reduction in SBP and $4.7 \mathrm{mmHg}$ of reduction in DBP in interventional group whereas control group showed $1 \mathrm{mmHg}$ of reduction in SBP and $1.8 \mathrm{mmHg}$ of reduction in DBP. Similar study conducted by Muhammad Amer et al. ${ }^{2}$ showed $10 \mathrm{mmHg}$ of reduction in SBP and $4.5 \mathrm{mmHg}$ of reduction in DBP in intervention group. Our present study has also demonstrated similar reduction in SBP and DBP in intervention group compared with those of control group. However the mean baseline SBP and DBP were significantly different in both the groups compared to previously published studies which had similar mean baseline SBP and DBP in both the groups. ${ }^{1-3}$ An initial high prevalence of non-adherence in interventional group (44.89\%) in our study could be a reason for greater reduction in blood pressure from baseline to final visit.

\begin{tabular}{|c|c|c|c|c|}
\hline Groups & $\begin{array}{l}\text { High n } \\
(\%)\end{array}$ & $\begin{array}{l}\text { Medium } \\
\mathrm{n}(\%)\end{array}$ & $\begin{array}{c}\text { Low n } \\
(\%)\end{array}$ & $p$ value $^{a}$ \\
\hline \multicolumn{5}{|l|}{$\begin{array}{c}\text { INTERVENTION } \\
n=49\end{array}$} \\
\hline Baseline Visit & $27(55.1)$ & $18(36.7)$ & $4(8.1)$ & $0.0011^{*}$ \\
\hline Final Visit & $43(87.7)$ & $6(12.2)$ & $0(0.0)$ & \\
\hline \multicolumn{5}{|l|}{ CONTROL $n=46$} \\
\hline Baseline Visit & $30(65.2)$ & $13(28.2)$ & $3(6.5)$ & 0.5150 \\
\hline Final Visit & $29(63.0)$ & $16(34.7)$ & $1(2.1)$ & \\
\hline
\end{tabular}

a- $p$ value obtained from chi square test *- Statistically significant.

\begin{tabular}{|c|c|c|c|c|}
\hline Groups & $\begin{array}{l}\text { High n } \\
(\%)\end{array}$ & $\begin{array}{l}\text { Medium } \\
\mathrm{n}(\%)\end{array}$ & $\begin{array}{l}\text { Low n } \\
(\%)\end{array}$ & $P$ value $^{a}$ \\
\hline \multicolumn{5}{|l|}{$\begin{array}{c}\text { INTERVENTION } \\
n=49\end{array}$} \\
\hline Baseline Visit & $21(42.8)$ & $22(44.8)$ & $6(12.2)$ & $<0.0001^{*}$ \\
\hline Final Visit & $44(89.7)$ & $5(10.2)$ & $0(0.0)$ & \\
\hline \multicolumn{5}{|l|}{ CONTROL $n=46$} \\
\hline Baseline Visit & $18(39.1)$ & $22(47.8)$ & $6(13.0)$ & 0.9046 \\
\hline Final Visit & $20(43.4)$ & $20(43.4)$ & $6(13.0)$ & \\
\hline
\end{tabular}

a- $P$ value obtained from chi square

*- Statistically significant.

Indian Journal of Pharmacy Practice, Vol 15, Issue 1, Jan-Mar, 2022
Findings of study conducted by Jose A. et al. ${ }^{9}$ showed $23.15 \mathrm{mmHg}$ reduction in SBP and $12.34 \mathrm{mmHg}$ in DBP which was highest compared to other studies and thought due to initial high prevalence of uncontrolled blood pressure $(80 \%)$ in study population. Similar findings of reduction in SBP and DBP in interventional group compared to control group have been reported by various studies. ${ }^{10-16}$ Findings reported by Sean Hennessy et al. ${ }^{17}$ was contradictory to our result which showed no effect or at best modest effect of intervention among hypertensive patients. Study published by Maxwell Anderegg, ${ }^{18}$ demonstrated that physician-pharmacist collaborative intervention was effective in reducing mean SBP and improving BP control in patient with uncontrolled hypertension.

Prevalence of non-adherence among hypertensive patient was found to be $54 \%$ in a study conducted by Arjun Balasubramanian et al. ${ }^{19}$ Similarly a study conducted at a healthcare facility in north India by Siraj Ahmad et al. ${ }^{20}$ showed prevalence of non-adherence among hypertensive patient to be $42.8 \%$. Study conducted by Prateek et al. ${ }^{21}$ have reported non-adherence to be $87 \%$ among hypertensive patients in rural area of Tamil Nadu. Our present study demonstrated prevalence of non-adherence to be $40 \%$. Most common reason for medication non-adherence was forgetfulness and carelessness among hypertensive patients. Lack of knowledge regarding disease condition and medications can lead to non-adherence. Attitude and behaviour of hypertensive patients changed after providing patient counselling and educational intervention. A significant change in adherence was observed at end of study in intervention group however no change was observed in control group which was similar with the study findings of Manuel morgado et al. ${ }^{12}$ that pharmacist intervention improved medication adherence to $74.5 \%$ in intervention group compared to control group with adherence rate of $57.6 \%$. It must be acknowledged that several other studies have also reported positive outcomes by change in medication adherence through providing pharmacist intervention. . $^{1,8,12,15}$

Our findings regarding hypertension related knowledge indicated that majority of patient were mindful of the normal range of blood pressure. Patients were aware regarding all the risk factors for developing hypertension, symptoms of low blood pressure, various complications of uncontrolled blood pressure and knew that medications alone cannot control the blood pressure. There were many patients with the belief that hypertension can be cured. Improved knowledge at the end of study in intervention group was result 
of PIL based patient counselling because the similar improvement was not seen in control group and thus pharmacist intervention were deemed to be effective in enhancing knowledge regarding various aspects of hypertension Patients with family member suffering from hypertension had better knowledge regarding the disease compared to patient with no family history of hypertension due to better awareness of disease condition. Study conducted by Muhammad Amer et ll $^{2}$ have also reported improvement in disease related knowledge in intervention group compared to control group. Michael Smith $e t$ al..$^{22}$ highlighted the need for increase disease state education in india. Study published by Rampamba et al..$^{23}$ demonstrated that majority of individual lacked hypertension related knowledge which was similar to findings reported in our study.

The effectiveness of pharmacist led intervention program was clearly evident from the outcomes, which in turn also changed the perceptions and practices. ${ }^{2}$ Hypertension related education to patients plays an important role in controlling blood pressure and improving medication adherence. Additionally, pharmacist intervention was recognized and appreciated by patients and healthcare providers in the current study. In addition to this patients also suggested to conducted education program at various community level to improve disease related outcomes.

Study only included patients who took antihypertensive medications at least for a month. Limited data on long term benefits of the counselling provided to the patients due to the short span of the study. Many potential patients were reluctant to participate due to continuous follow up required in the study.

The positive outcomes of study will be helpful in involving pharmacist along with physician care for commonly occurring chronic illnesses. The study can further be expanded in future regarding its effect on vulnerable group like pregnant women and those with end stage liver disease and end stage renal disease. Provision of proper counselling to the patients with chronic disease will be helpful in better management of their existing condition.

\section{CONCLUSION}

The study highlighted that education and patient counselling based intervention improved blood pressure and medication adherence in hypertensive patients. The intervention was helpful in enhancing knowledge and creating awareness regarding disease and medication.
Similarly our study made an overall positive impact on patients regarding pharmacist. Furthermore, this study report also addresses the pharmacist plays an important role in effective participation in management of hypertension as an essential part of traditional physician based care which in addition will help in improving traditional framework of Indian healthcare system.

\section{ACKNOWLEDGEMENT}

Authors acknowledge the patients for participation in this study and staff of hospital for their support in successfully accomplishing the study. We would like to appreciation Dr Darshan Pandya and Dr Maulesh Tailor for allowing us to carryout for research work in their hospital.

\section{CONFLICT OF INTEREST}

The authors declare that there is no conflict of interest.

\section{ABBREVIATIONS}

PIL: Patient Information Leaflet; MMAS: Morisky Medication Adherence Scale; SBP: Systolic Blood Pressure; DBP: Diastolic Blood Pressure; CRF: Case Record Form.

\section{SUMMARY}

The study was conducted to evaluate the effectiveness of patient counselling based intervention on blood pressure, medication adherence and knowledge in hypertensive patients. It was a prospective, multi-centric, randomized controlled interventional study conducted for period of 4 months at 2 outpatient's clinics in Gandhinagar city, Gujarat. Enrolled patients were randomized into 2 groups (Intervention and Control) using simple randomization technique with allocation ratio of 1:1. Morisky Medication Adherence Scale (MMAS-4), self-designed Case Record Form (CRF) and self-designed knowledge questionnaire were utilised for data collection. Each patient was followed for 2 months with a total of 4 follow-ups each 15 days apart. Patient counselling and education was provided using patient information leaflet (PIL) in interventional group. A total of 105 patients were randomized into two groups. The result revealed significant reduction in systolic blood pressure (SBP) and in Diastolic Blood Pressure (DBP) in interventional group from baseline to final visit whereas no significant difference was seen in control group. Interventional group showed significant change in 
medication adherence as well as in hypertension related knowledge wherein control group showed no significant change at the end of study. Hence, patient counselling based intervention using PIL improved blood pressure and medication adherence in hypertensive patients. The intervention was helpful in enhancing knowledge and creating awareness regarding disease and medication.

\section{REFERENCES}

1. Zhao P. Effect of clinical pharmacists pharmaceutical care intervention to control hypertensive outpatients in China. Afr J Pharm Pharmacol. 2012;6(1):48-56. doi: 10.5897/AJPP11.633.

2. Amer M, Rahman N, Nazir SR, Raza A, Riaz H, Sultana M, Sadeeqa S. Impact of pharmacist's intervention on disease related knowledge, medication adherence, HRQoL and control of blood pressure among hypertensive patients. Pak J Pharm Sci. 2018;31(6);(Suppl;(Suppl:2607-16. PMID 30587468.

3. Wang J, Wu J, Yang J, Zhuang Y, Chen J, Qian W, Tian J, Chen X, She D, Peng F. Effects of pharmaceutical care interventions on blood pressure and medication adherence of patients with primary hypertension in China. Clin Res Regul Aff. 2011;28(1):1-6. doi: 10.3109/10601333.2010.539230.

4. Pawar S, Lokhande KD, Padma SO, Diwan AR. Effect of pharmacist mediated patient counseling in hypertensive patients in terms of knowledge, compliance and lifestyle modification. Int J Pharm Pharm Sci. 2014;6(4):277-81.

5. Mathew A, Paluri V, Venkateswaramurthy N. A study on impact of clinical pharmacist interventions on relationship between treatment satisfaction and medication adherence in hypertensive patients. J Pharm Sci Res. 2016;8(4):190.

6. Ramanath KV, Balaji DB, Nagakishore Ch, Kumar SM, Bhanuprakash M. A study on impact of clinical pharmacist interventions on medication adherence and quality of life in rural hypertensive patients. J Young Pharm. 2012;4(2):95-100. doi: 10.4103/0975-1483.96623, PMID 22754261.

7. Sahoo SK, Preeti PS, Biswas D. Adherence to anti-hypertensive drugs: A clinic based study among geriatric hypertensive patients in Rural, India. Natl J Commun Med. 2018;9(4):250-4.

8. Saleem F, Hassali MA, Shafie AA, UI Haq N, Farooqui M, Aljadhay H, Ahmad FU. Pharmacist intervention in improving hypertension-related knowledge, treatment medication adherence and health-related quality of life: a non-clinical randomized controlled trial. Health Expect. 2015;18(5):1270-81. doi: 10.1111/hex.12101, PMID 23786500.

9. Garçao JA, Cabrita J. Evaluation of a pharmaceutical care program for hypertensive patients in rural Portugal. J Am Pharm Assoc (Wash). 2002;42(6) (6):858-64. doi: 10.1331/108658002762063691, PMID 12482009.

10. Chabot I, Moisan J, Grégoire JP, Milot A. Pharmacist intervention program for control of hypertension. Ann Pharmacother. 2003 Sep;37(9):1186-93. doi: 10.1345/aph.1C267, PMID 12921497.

11. Sookaneknun P, Richards RM, Sanguansermsri J, Teerasut C. Pharmacist involvement in primary care improves hypertensive patient clinical outcomes.
Ann Pharmacother. 2004;38(12):2023-8. doi: 10.1345/aph.1D605, PMID 15522983.

12. Borenstein JE, Graber G, Saltiel E, Wallace J, Ryu S, Archi J, Deutsch S, Weingarten SR. Physician-pharmacist comanagement of hypertension: a randomized, comparative trial. Pharmacotherapy. 2003;23(2):209-16. doi: 10.1592/phco.23.2.209.32096, PMID 12587810.

13. Hennessy S, Leonard CE, Yang W, Kimmel SE, Townsend RR, Wasserstein AG, Ten Have TR, Bilker WB. Effectiveness of a two-part educational intervention to improve hypertension control: a cluster-randomized trial. Pharmacotherapy. 2006;26(9):1342-7. doi: 10.1592/phco.26.9.1342, PMID 16945057.

14. Bajorek B, Lemay KS, Magin P, Roberts C, Krass I, Armour CL. Implementation and evaluation of a pharmacist-led hypertension management service in primary care: outcomes and methodological challenges. Pharm Pract (Granada). 2016;14(2):723-. doi: 10.18549/PharmPract.2016.02.723, PMID 27382427.

15. Parker CP, Cunningham CL, Carter BL, Vander Weg MW, Richardson KK, Rosenthal GE. A mixed-method approach to evaluate a pharmacist intervention for veterans with hypertension. J Clin Hypertens (Greenwich). 2014;16(2):133-40. doi: 10.1111/jch.12250, PMID 24588813.

16. Edelman D, Fredrickson SK, Melnyk SD, Coffman CJ, Jeffreys AS, Datta S, Jackson GL, Harris AC, Hamilton NS, Stewart H, Stein J, Weinberger M. Medical clinics versus usual care for patients with both diabetes and hypertension: a randomized trial. Ann Intern Med. 2010;152(11):689-96. doi: 10.7326/00034819-152-11-201006010-00001, PMID 20513826.

17. Anderegg MD, Gums TH, Uribe L, MacLaughlin EJ, Hoehns J, Bazaldua OV, Ives TJ, Hahn DL, Coffey CS, Carter BL. Pharmacist intervention for blood pressure control in patients with diabetes and/or Chronic Kidney Disease. Pharmacother J Hum Pharmacol Drug Ther. 2018;38(3):309-18. doi: 10.1002/ phar.2083.

18. Balasubramanian A, Nair SS, Rakesh PS, Leelamoni K. Adherence to treatment among hypertensives of rural Kerala, India. J Fam Med Prim Care. 2018;7(1):64-9. doi: 10.4103/jfmpc.jfmpc_423_16, PMID 29915735.

19. Ahmed S. Assessment of adherence to antihypertensive treatment among patients attending a health care facility in North India. Int $\mathrm{J}$ Res Med. 2015;4(1):117-24.

20. Shrivastava PS, Shrivastava SR, S M. A community-based study to assess the treatment adherence and its determinants among hypertensive patients residing in a rural area of Kancheepuram district, Tamil nadu. Int J Med Sci Public Health. 2017;6(9):1386-94. doi: 10.5455/ijmsph.2017.0409630062017.

21. Morgado M, Rolo S, Castelo-Branco M. Pharmacist intervention program to enhance hypertension control: a randomised controlled trial. Int J Clin Pharm. 2011;33(1):132-40. doi: 10.1007/s11096-010-9474-x, PMID 21365405.

22. Smith MT, Monahan MP, Nelson P, Moruzzi M, DeLucenay AJ, Birnie CR. Elevated blood pressure in the developing world: a role for clinical pharmacists. Int J Pharm Pract. 2018;26(4):334-40. doi: 10.1111/ijpp.12398, PMID 28925056.

23. Rampamba EM, Meyer JC, Helberg E, Godman B. Knowledge of hypertension and its management among hypertensive patients on chronic medicines at primary health care public sector facilities in South Africa; findings and implications. Expert Rev Cardiovasc Ther. 2017;15(8):639-47. doi: 10.1080/14779072.2017.1356228, PMID 28712328. 